\title{
Bis(pentamethylcyclopentadienyl)methanol: Synthese, Struktur und Derivatisierung
}

\author{
Peter Jutzi*, Andreas Mix, Tanja Lindermeier, Hans-Georg Stammler und Beate Neumann \\ Fakultät für Chemie der Universität Bielefeld, \\ Universitätsstraße 25, 33615 Bielefeld
}

Eingegangen am 21. April 1993

Key Words: Bis(pentamethylcyclopentadienyl)methanol / Diels-Alder reaction, intramolecular / Chloroformate, bis(pentamethylcyclopentadienyl)methyl / Tosylate, bis(pentamethylcyclopentadienyl)methyl

\begin{abstract}
Bis(pentamethylcyclopentadienyl)methanol: Synthesis, Structure, and Derivatization
Bis(pentamethylcyclopentadienyl) ketone (1) reacts at low temperatures with $\mathrm{LiAlH}_{4}$ to form in good yields (pentamethylcyclopentadienyl)methanol (2) and its corresponding intramolecular Diels-Alder product 3 in a ca. 2:1 ratio. The alcohol 2 is separated from the tetracyclic compound 3 by crystallisation at $-70^{\circ} \mathrm{C}$ from hexane/pyridine as pyridine adduct. The pyridine-free alcohol 2 is available by repeated dissolving of the adduct in hexane and removal of the volatile compo-

cycloaddition even at room temperature with formation of 3 A derivatization of $\mathbf{2}$ is possible by deprotonation with alkyllithium compounds, which leads to the alcoholate 4 , and subsequent treatment with phosgen and tosyl chloride, thus affording the chloroformate 6 and the tosylate 7, respectively, in good yields. The thermolabile tosylate 7 decomposes to pentamethylbenzene or, under basic conditions, to the pentacyclic compound $\mathbf{7}$.
\end{abstract} nents in vacuo. The alcohol tends to intramolecular $[4+2]$
Hauptgruppenelement-Verbindungen mit zwei $\sigma$-gebundenen Pentamethylcyclopentadienyl $\left(\mathrm{Cp}^{*}\right)$-Liganden sind generell als Vorstufen für Verbindungen interessant, in denen das entsprechende Zentralatom durch $\pi$-Komplexierung und Ausbildung einer sandwichartigen Struktur stabilisiert wird. Das Potential des C $\mathrm{p}^{*}$-Liganden zeigt sich besonders eindrucksvoll bei den Gruppe-14-Elementen. So konnten für alle Homologen des Kohlenstoffs Komplexe mit formal zweiwertigem Zentralatom dargestellt werden ${ }^{[1-4]}$. Um zu überprüfen, inwieweit das Konzept der $\pi$-Stabilisierung auch auf Kohlenstoff selbst übertragbar ist, untersuchen wir zur Zeit Pentamethylcyclopentadienyl-substituicrte Kohlenstoff-Verbindungen ${ }^{15.61}$. Insbesondere $\mathrm{C}_{1}$-Verbindungen mit zwei $\mathrm{Cp}^{*}$-Liganden sind für uns in dicsem Zusammenhang von Interesse.

\section{Ergebnisse}

Vor kurzem berichteten wir über die Synthese der ersten Kohlenstoff-Verbindungen, die zwei $\mathrm{Cp}^{*}$-Liganden an einer zentralen $\mathrm{sp}^{2}$-hybridisierten $\mathrm{C}_{1}$-Einheit tragen ${ }^{\left[{ }^{6}\right]}$. Im Gegensatz zu den bisher untersuchten zweifach $\mathrm{Cp}^{*}$-substituierten Hauptgruppenelement-Verbindungen zeigen die Kohlenstoff-Derivate bereits bei Raumtemperatur eine ausgesprochene Tendenz zur intramolekularen Diels-Alder-Reaktion. Verantwortlich für die Cyclisierungstendenz dieser Verbindungen ist das Vorliegen einer Konformation mit kurzem Abstand zwischen den relevanten $\pi$-Systemen. Es stellt sich die Frage, ob $\mathrm{C}_{1}$-Verbindungen mit $\mathrm{sp}^{3}$-hybridisiertem Zentralatom und zwei $\mathrm{Cp}^{*}$-Liganden in bezug auf die intramolekulare $[4+2]$-Cycloaddition unter Normalbedingungen überhaupt stabil sind, da die zu erwartende Verringe- rung des Bindungswinkels am zentralen C-Atom beim Übergang vom $\mathrm{sp}^{2}-$ zum $\mathrm{sp}^{3}$-Hybrid zu einer weiteren Verkürzung der Distanz der $\mathrm{Cp}^{*}$-Systeme und damit zu einer Zunahme der Diels-Alder-Aktivität führen sollte. Es ist uns nun gelungen, derartige Verbindungen darzustellen. Setzt man das zweifach $\mathrm{Cp}^{*}$-substituierte Keton $1^{\left[{ }^{6]}\right.}$ mit $\mathrm{LiAlH}_{4}$ bei $-30^{\circ} \mathrm{C}$ in THF um, so erhält man nach einer Reaktionszeit von $12 \mathrm{~h}$ und anschließender hydrolytischer Aufarbeitung (Pentamethylcyclopentadienyl)methanol (2) im Gemisch mit dessen Diels-Alder-Produkt 3. Die Alkohole 2 und $\mathbf{3}$ fallen dabei als farblose Pulver im Verhältnis von ca. 2:1 an (Gl. 1).

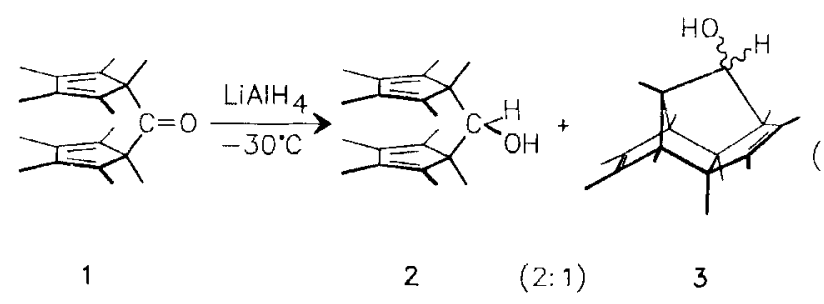

Das Vorliegen der Verbindungen 2 und 3 ist durch spektroskopische und elementaranalytische Daten gesichert. Beide Verbindungen sind sehr gut löslich in allen gängigen organischen Solvenzien. Eine Trennung, beispielsweise durch fraktionierte Kristallisation oder Chromatographie, bereitet enorme Schwierigkeiten. Bei Zugabe von Pyridin zu einer Lösung des Produktgemisches kristallisiert der Alkohol 2 bei $-70^{\circ} \mathrm{C}$ als 1:1-Addukt mit der Base in farblosen Rauten. Die Base läßt sich anschließend durch wiederholtes Lösen des Adduktes in Hexan und Abdestillieren der flüchtigen Bestandteile im Vakuum entfernen. Verbindung 2 ist 
auf diese Weise pyridinfrei in etwa 70proz. Ausbeute zugänglich.

Der Alkohol 2 neigt zur intramolekularen Diels-AlderReaktion. Bei $-70^{\circ} \mathrm{C}$ kann 2 über mehrere Monate gelagert werden. Bei Raumtemperatur ist der Alkohol nur kurzfristig stabil; innerhalb von $12 \mathrm{~h}$ wandelt er sich sowohl in Lösung als auch im Festkörper in den Tetracyclus 3 um (Gl. 2). Verbindung 3 fällt dabei als Diastereomerengemisch an.

Vom Pyridin-Addukt des Alkohols 2 haben wir eine Röntgenstrukturanalyse durchgeführt (Abb. 1, Tab. 1).

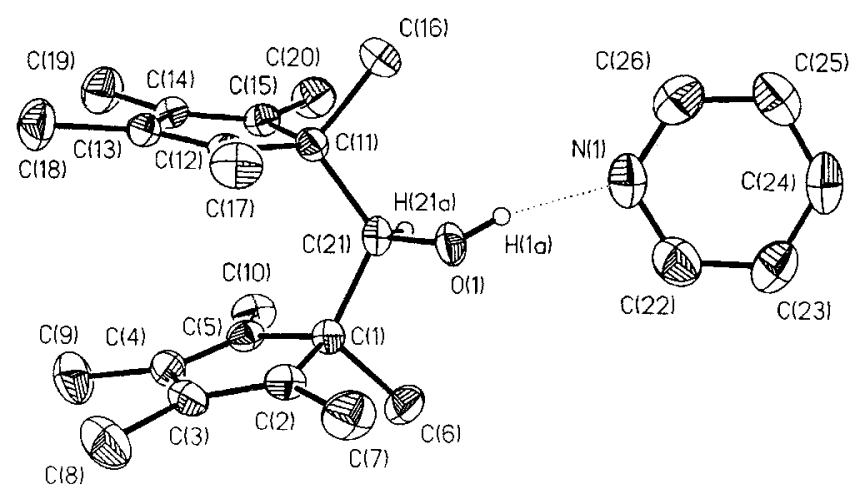

Abb. 1. Molekülstruktur des Adduktes aus 2 und Pyridin. Bindungslängen $[\AA]$ und -winkel $\left[{ }^{\circ}\right.$; $\mathrm{C}(1)-\mathrm{C}(2)$ 1.519(5), $\mathrm{C}(1)-\mathrm{C}(21)$ $1.558(4), \mathrm{C}(2)-\mathrm{C}(3) 1.332(4), \mathrm{C}(3)-\mathrm{C}(4) 1.470(5), \mathrm{C}(21)-\mathrm{O}(1) 1.407(4)$, $\mathrm{O}(1)-\mathrm{H}(1 \mathrm{a}) 0.846$ - $\mathrm{C}(1)-\mathrm{C}(21)-\mathrm{C}(11) 119.5(2), \mathrm{O}(1)-\mathrm{C}(21)-\mathrm{C}(1)$ $106.7(2), \mathrm{O}(1)-\mathrm{C}(21)-\mathrm{C}(11) 111.4(3)$

Tab. 1. Daten zur Kristallstruktur von $2 \cdot$ Pyridin $^{[181}$

Summenformel $\mathrm{C}_{26} \mathrm{H}_{37} \mathrm{NO} ;$ Molmasse $379.6 ;$ Zelldaten $[\AA]$ : $a=8.100(2), b=8.702(3), c=17.371(4), \alpha=77.05(2)^{\circ}, \beta=83.15(2)^{\circ}$, $\gamma=71.59(2)^{\circ}$; Zellvolumen $\left[\AA^{3}\right] 1130.6(5)$; Kristallsystem triklin; Raumgruppe $P \overline{1} ; d_{\mathrm{c}}=1.115 \mathrm{~g} / \mathrm{cm}^{3} ; Z=2 ; F(000)=416$; Strahlung: Mo- $K_{\infty}, \lambda=0.71073 \AA ; \mu=0.062 \mathrm{~mm}^{-1} ; 2 \Theta \mathrm{min} / \max =3.0 / 48.0$; Meßtemperatur $163 \mathrm{~K}$, gemessene Reflexe 4753; beobachtete Reflexe $2949[F>4.0 \sigma(F)]$; Programm zur Strukturlösung und -verfeinerung SHELXTL Plus; Zahl der verfeinerten Parameter 262; $R=0.062, R_{\mathrm{w}}=0.057 ; w^{-1}=\sigma^{2}(F)$

Die Elementarzelle enthält zwei Einheiten des Adduktes. Das Pyridin ist über eine schwache Wasserstoffbrücken-Bindung an die Hydroxy-Einheit von 2 koordiniert (N-H-Abstand $2.00 \AA$ ). Die Struktur ist bezüglich des Methin-Protons und der Hydroxy-Gruppe am zentralen KohlenstoffAtom $\mathrm{C}(21)$ fehlgeordnet. Abb. 1 repräsentiert 79\% der Moleküle im Kristall. Die Cyclopentadienyl-Einheiten in $\mathbf{2}$ sind planar; die Ebenen-Normalen schließen einen Winkel von $161.7^{\circ}$ ein. Während die Werte für die Bindungslängen jeweils in durchaus typischen Bereichen liegen, zeigen sich bei den Bindungswinkeln Auffälligkeiten. So ist die Geometrie am zentralen Kohlenstoffatom $C(21)$ durch den großen sterischen Anspruch der beiden PentamethylcyclopentadienylLiganden stark verzerrt. Der Winkel $C p^{*}-C(21)-C p^{*}$ ist mit $119.5^{\circ}$ relativ zum idealen Tetraederwinkel extrem aufgeweitet. Der Abstand zwischen den Mittelpunkten der $\mathrm{Cp}^{*}$ Liganden beträgt $3.22 \AA$. Offensichtlich ist eine weitere Annäherung zweier $\mathrm{Cp}$-Fragmente nicht möglich und somit im Vergleich $\mathrm{zu}$ anderen Verbindungen mit zwei $\mathrm{Cp}^{*}$ -
Liganden ${ }^{[6,7]}$ in dieser Verbindung ein Grenzwert erreicht. Die Minimierung der sterischen Wechselwirkung zwischen Proton und Hydroxy-Gruppe an $\mathrm{C}(21)$ einerseits und den allylständigen Methylgruppen der beiden Ringsysteme andererseits erlaubt in 2 lediglich eine Torsion der beiden $C \mathrm{p}^{*}$ Liganden von $24.2^{\circ}$. Damit sind die Ringsysteme in dieser Verbindung deutlich geringer gegeneinander verdreht als in der von uns untersuchten Keto- und Thioketo-Verbindung ${ }^{[6]}$. Dennoch ist dieser vergleichsweise kleine Torsionswinkel für eine weitestgehend gestaffelte Anordnung der übrigen ringständigen Methylgruppen ausreichend.

Die Kristallstruktur zeigt auch den Grund für die mäßig gesteigerte intramolekulare Diels-Alder-Aktivität des Alkohols 2 relativ zu den zweifach $\mathrm{Cp}^{*}$-substituierten Kohlenstoff-Verbindungen mit $\mathrm{sp}^{2}$-hybridisiertem Zentralatom. Die sehr geringe Distanz zwischen beiden $\mathrm{Cp}^{*}$-Liganden und die aus sterischen Gründen erzwungene deutliche Aufweitung des Bindungswinkels an $\mathrm{C}(21)$ sollten eine wesentlich drastischere Steigerung der Cycloadditions-Tendenz von 2 bewirken. Diese Effekte werden aber teilweise durch die für eine Diels-Alder-Reaktion ungünstige Geometrie der Doppelbindungen kompensiert. Durch den beobachteten Torsionswinkel zwischen den beiden $\mathrm{Me}_{5} \mathrm{C}_{5}$-Liganden werden die $\pi$-Systeme der $\mathrm{Cp}^{*}$-Einheiten nahezu parallel übereinander angeordnet. Wie in Abb. 2 gezeigt, hat dies deutlich unterschiedliche Distanzen zwischen den für die $\mathrm{Cy}$ cloaddition relevanten Kohlenstoffatomen zur Folge $\left[d_{\mathrm{C}(2)-\mathrm{C}(12)}=3.17, d_{\mathrm{C}(5)-\mathrm{C}(13)}=3.54 \AA\right]$. Durch die vorliegende Geometrie wird ein Ringschluß offensichtlich erschwert.

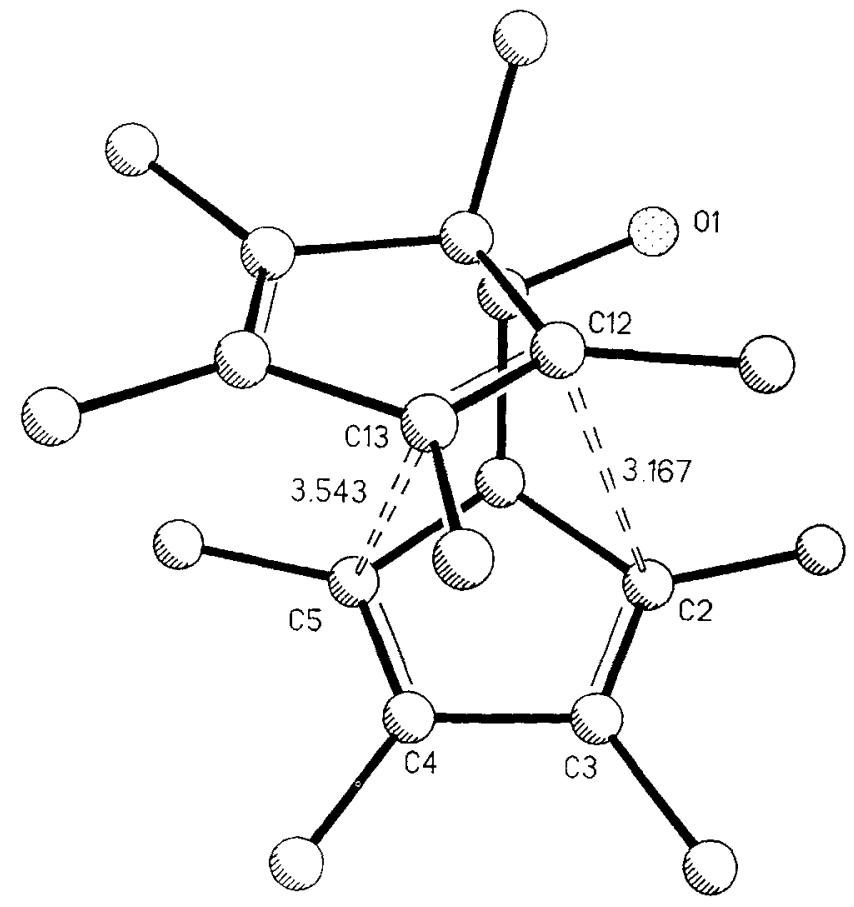

Abb. 2. Orientierung der $\mathrm{Cp}^{*}$-Einheiten in 2

\section{Chemisches Verhalten von 2}

Neben der ausgeprägten Tendenz zur intramolekularen $[4+2]$-Cycloaddition zeigt der Alkohol weitere Besonder- 
heiten. So ist beispielsweise eine Derivatisierung der Hydroxy-Funktion durch Umsetzung mit elektrophilen Reagenzien, wie zum Beispiel Sulfonsäurechloriden oder mit Chlortrimethylsilan, nicht möglich. Verantwortlich hierfür ist zweifellos der enorme sterische Einfluß der beiden Pentamethylcyclopentadienyl-Liganden, der einen Angriff am Sauerstoffatom erschwert. Der Alkohol 2 ist allerdings mit starken Basen, wie zum Beispiel Alkyllithium-Verbindungen, deprotonierbar. Das entstehende Alkoholat $\mathbf{4}$ ist selbst bei tieferen Temperaturen ungewöhnlich instabil. Es zersetzt sich auch in vergleichsweise inerten aprotischen Lösungsmitteln wie Hexan oder THF unter Abspaltung von Pentamethylcyclopentadienyllithium und Bildung von 5-Formylpentamethylcyclopentadien 5. Beide Produkte lassen sich spektroskopisch eindeutig charakterisieren ${ }^{[8,9]}$. Überraschenderweise erweist sich das Alkoholat 4 in Diethylether als Solvens bei $-30^{\circ} \mathrm{C}$ als so stabil, daß eine Derivatisierung mit elektrophilen Reagenzien möglich ist. Setzt man das Alkoholat mit einer äquimolaren Menge Phosgen um, erhält man in guter Ausbeute den entsprechenden Chlorameisensäureester 6 (G1. 3). Die Verbindung ist sehr gut löslich in gängigen organischen Solvenzien.

Aus einer konzentrierten Hexan-Lösung kristallisiert der Ester bei tiefen Temperaturen in farblosen, dünnen Plättchen. 6 ist thermisch nicht sonderlich beständig. Bereits bei Raumtemperatur zersetzt sich die Verbindung langsam. Bei der Temperatur des Schmelzpunktes tritt schnelle Zersetzung ein.

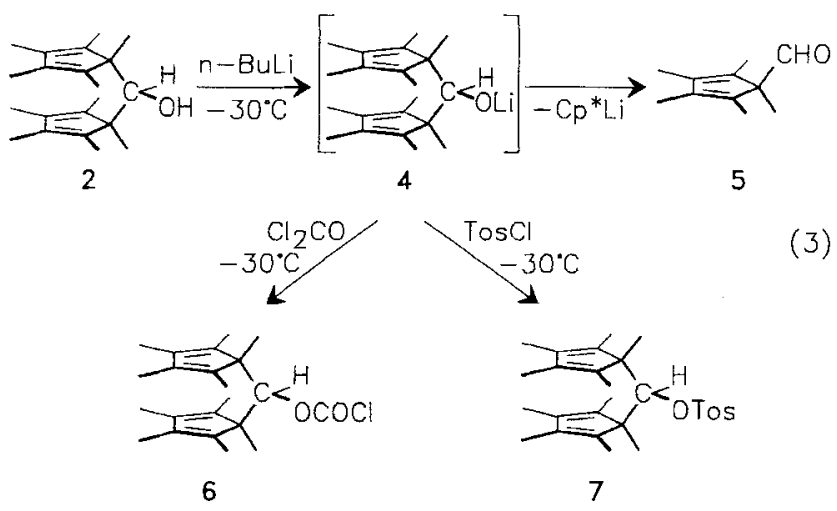

Von 6 haben wir eine Röntgenstrukturanalyse durchgeführt (Abb. 3, Tab. 2).

Die Elementarzelle enthält acht Moleküleinheiten. Innerhalb der $\mathrm{Cp}^{*} \mathrm{CC} \mathrm{p}^{*}$-Struktureinheit sind Ähnlichkeiten zur Struktur von 2 . Pyridin zu beobachten. Der Cp*CCp*Winkel beträgt $121.1^{\circ}$ und ist gegenüber 2 - Pyridin allerdings noch stärker aufgeweitet; der Abstand zwischen den Mittelpunkten des Cp*-Liganden ist gleich (3.22 $\AA$ ). Die Torsion der beiden planaren $\mathrm{Cp}^{*}$-Liganden ist mit 22.2 vergleichsweise kleiner. Auffällig ist der im Vergleich $\mathrm{zu} 2$ Pyridin $(1.407 \AA)$ merklich vergrößerte C-O-Abstand (1.484 A) in 6 .

Dic Reaktion von 4 mit 4-Toluolsulfonylchlorid führt zum entsprechenden Tosylat 7 (Gl. 3), welches als farbloses Pulver in ca. 80proz. Ausbeute im Gemisch mit Lithiumchlorid anfällt. Im Gegensatz zum Chlorformiat 6 ist das
Tosylat 7 sowohl in Hexan und THF als auch in aromatischen Solvenzien äußerst schlecht Iöslich. Daher ist eine vollständige Abtrennung des Produktes vom Lithium-Salz nicht möglich. 7 ist aber durch NMR-spektroskopische und massenspektrometrische Daten eindeutig charakterisiert (Tab. 3).

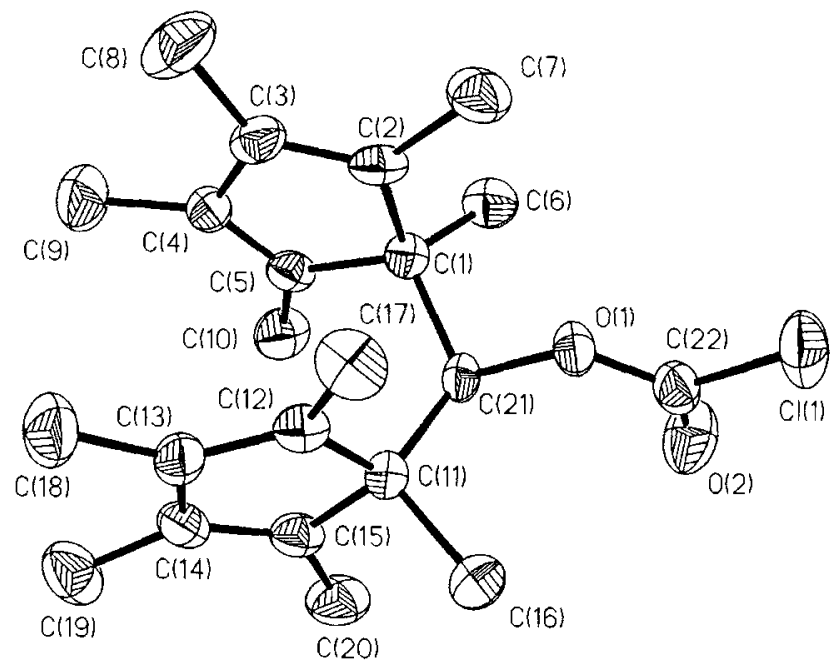

Abb. 3. Molekülstruktur von 6. Bindungslängen $[\AA]$ und -winkel ["]: $C(1)-C(2) \quad 1.522(5), C(2)-C(3) \quad 1.332(5), C(3)-C(4) 1.471(5)$ $\mathrm{C}(1)-\mathrm{C}(21)$ 1.556(5), $\mathrm{C}(21)-\mathrm{O}(1)$ 1.484(4), $\mathrm{C}(22)-\mathrm{O}(1) 1.313(5)$ $\mathrm{C}(22)-\mathrm{O}(2)$ 1.176(5), $\mathrm{C}(22)-\mathrm{Cl}(1) 1.752(4) .-\mathrm{C}(1)-\mathrm{C}(21)-\mathrm{C}(11)$ 121.1(3), $\mathrm{C}(1)-\mathrm{C}(21)-\mathrm{O}(1)$ 105.1(3), $\mathrm{C}(11)-\mathrm{C}(21)-\mathrm{O}(1)$ 106.9(3), $\mathrm{O}(1)-\mathrm{C}(22)-\mathrm{Cl}(1) \quad 107.0(3), \quad \mathrm{O}(1)-\mathrm{C}(22)-\mathrm{O}(2) \quad 130.2(4), \quad \mathrm{O}(2)-$ $\mathrm{C}(22)-\mathrm{Cl}(1) 122.9(4)$

Tab. 2. Daten zur Kristallstruktur von $\mathbf{6}^{[18]}$

Summenformel $\mathrm{C}_{22} \mathrm{H}_{31} \mathrm{ClO}_{2} ;$ Molmasse 362.92 ; Zelldaten $(\AA)$ : $a=15.160(3), b=12.896(3), c=20.905(5), \alpha=\beta=\gamma=90^{\circ}$; Zellvolumen $\left[\AA^{3}\right]$ : 4087(2); Kristallsystem orthorhombisch; Raumgruppe Pbca; $d_{\mathrm{c}}=1.180 \mathrm{~g} / \mathrm{cm}^{3} ; Z=8 ; F(000)=1568$; Strahlung: Mo- $K_{\mathrm{z}}$, $\lambda=0.71073 \AA ; \mu=0.199 \mathrm{~mm}^{-1} ; 2 \Theta \mathrm{min} / \mathrm{max}=3.0 / 50.0^{\circ} ;$ Meßtemperatur $173 \mathrm{~K}$; gemessene Reflexe 4073; Programme zur Strukturlösung und -verfeinerung: SHELXS-86 und SHELXL-93; Zahl der vcrfeinerten Parameter 236; $R=0.0724$ für 1837 Reflexe mit $[F>$ $4.0 \sigma(F)]$

Die Thermolabilität des Tosylats 7 ist im Vergleich zum Chlorformiat 6 noch extremer. Bei Raumtemperatur zersetzt sich die Verbindung innerhalb von wenigen Minuten voll-

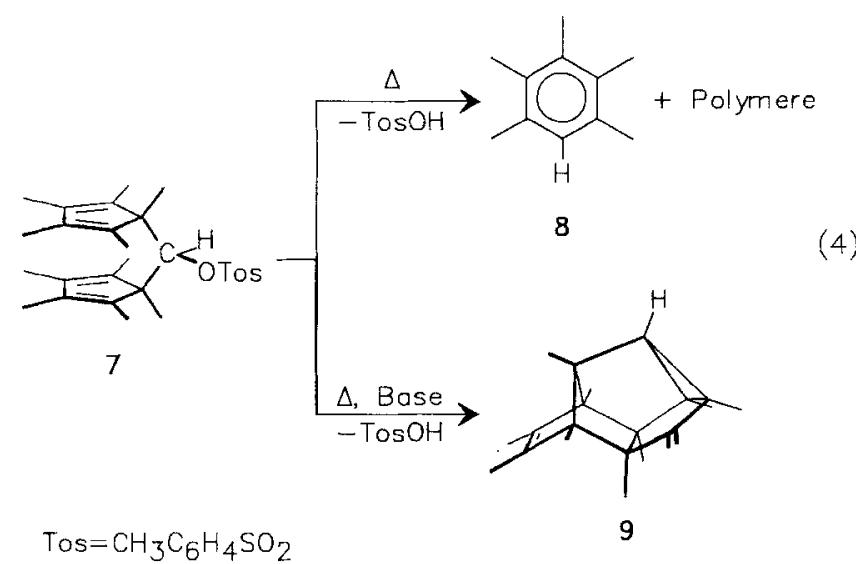


ständig, wobei sich ${ }^{1} \mathrm{H}$-NMR-spektroskopisch im Reaktionsgemisch neben 4-Toluolsulfonsäure nur Pentamethylbenzol (8) eindeutig nachweisen läßt (Gl. 4). Neben den Resonanzen für 8 und die Säure beobachtet man eine Vielzahl von Signalen im Bereich zwischen $\delta=1$ und 2. Dieses Erscheinungsbild ist typisch für polymere Produkte, welche aus der Zersetzung von $\mathrm{Cp}^{*}$-Einheiten resultieren ${ }^{[10]}$. Fängt man beim thermischen Zerfall von 7 die freiwerdende Säure jedoch mit einer Base ab, wie zum Beispiel mit Pyridin oder mit wäBriger Natriumhydrogencarbonat-Lösung, so erhält man als Reaktionsprodukt den pentacyclischen Kohlenwasserstoff 9 in quantitativer Ausbeute als blaßgelbes Pulver (Gl. 4) ${ }^{[11]}$.

Die Abspaltung einer Säure HX aus strukturell analogen einfach $\mathrm{Cp}^{*}$-substituierten $\mathrm{C}_{1}$-Verbindungen vom Typ $\mathbf{1 0}$ ist bereits beschrieben worden. De Vries ${ }^{[12,13]}$ und Winstein et al. ${ }^{[14]}$ postulieren als ersten Reaktionsschritt die heterolytische Spaltung der C-X-Bindung, die letztlich zu Carbokationen vom Typ 12 mit Bicyclo[3.1.0]hexen-Struktur führt (Gl. 5). In diesen Kationen sind die Protonen der Methylgruppen in 2- und 4-Stellung aktiviert; entsprechend werden diese Kationen in Gegenwart einer Base sofort zu Homofulvenen des Typs 13 deprotoniert ${ }^{[15]}$. In Abwesenheit einer Base lagern sich Kationen des Typs 12 oberhalb von $-30^{\circ} \mathrm{C}$ in die entsprechenden Cyclohexadienyl-Kationen $14 \mathrm{um}$, welche durch Abspaltung eines Protons und Aromatisierung Benzol-Derivate 15 bilden ${ }^{[14,15,16]}$.

Für die Zersetzung des Tosylates 7 favorisieren wir den von de Vries und Winstein vorgeschlagenen Reaktionsweg. Wir vermuten, daß im ersten Reaktionsschritt eine thermisch initiierte heterolytische Spaltung der extrem labilen C-X-Bindung zu einem Carbokation vom Typ 12 stattfindet ${ }^{[12]}$. Dieses Kation lagert sich in Abwesenheit einer Base sofort in das dem Typ 14 entsprechende HexadienylKation 16 um (Gl. 6).

Das Kation 16 kann sich jedoch nicht auf üblichem Wege durch Abspaltung von $\mathrm{H}^{+}$stabilisieren, da die Bildung eines

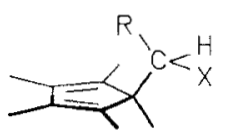

10

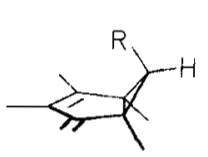

13
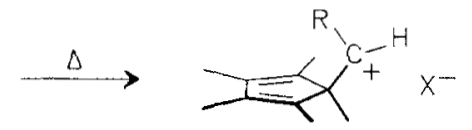

11

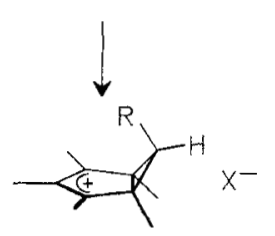

12
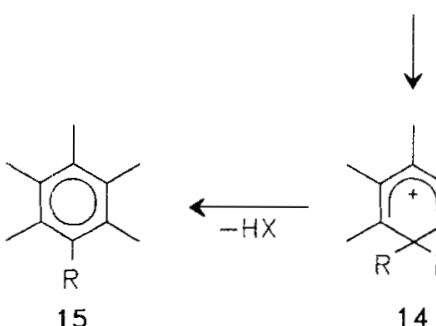

15
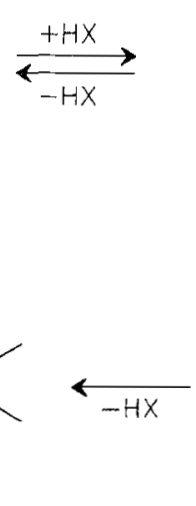

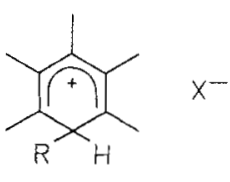

14
$\mathrm{Cp}^{*}$-substituierten Benzol-Derivates aufgrund sterischer Überfrachtung nicht möglich ist ${ }^{[10]}$. Stattdessen stabilisiert sich 16 unter Abspaltung von Pentamethylcyclopentadienyltosylat, $\mathrm{Cp} \mathrm{p}^{*} \mathrm{OTos}$, und Bildung von Pentamethylbenzol (8) (Gl. 6). Das Cp*OTos-Molekül zersetzt sich unter Polymerenbildung ${ }^{[17]}$.

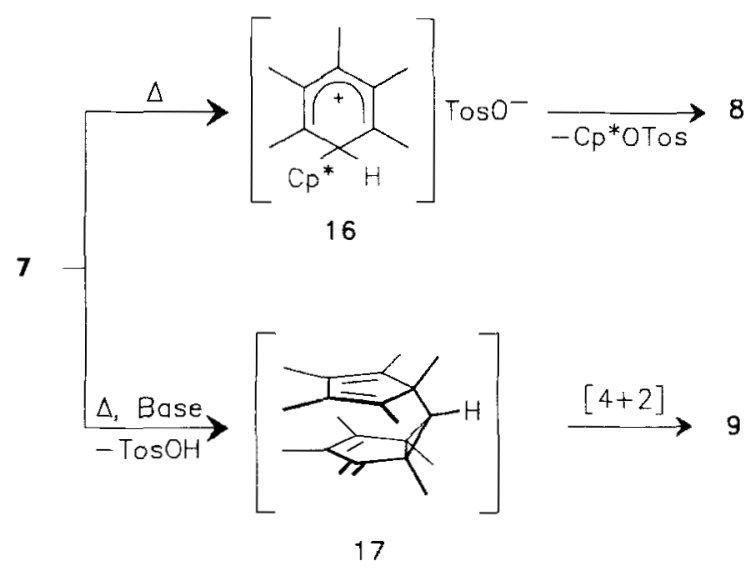

In Gegenwart einer Base wird 4-Toluolsulfonsäure aus dem Gleichgewicht [Gl. (5): $12 \rightleftarrows 13$ ] unter Bildung des Homofulvens 17 entfernt. In Verbindung 17 ist nun die geometrische Anordnung von endocyclischer Doppelbindung der bicyclischen Moleküleinheit und Diensystem des $\mathrm{Cp}^{*}$ Liganden für eine intramolekulare Diels-Alder-Reaktion derart günstig, da $B$ diese Verbindung selbst bei niedrigen Temperaturen sofort zum Endprodukt 9 cyclisiert.

Das Reaktionsverhalten des Tosylates 7 hat Konsequenzen für die weitere Strategie zur Synthese einer zweifach Cp*-substituierten Kohlenstoff(II)-Verbindung. Der Alkohol 2 ist für nachfolgende Eliminierungsreaktionen so zu derivatisieren, daß kationische Zwischenstufen (Typ 11 bzw. 12) vermieden werden.

\section{NMR-Spektren}

In den Verbindungen 2, 6 und 7 sind die $\mathrm{C}-\mathrm{CH}_{3}$-Gruppen in den Positionen 1 und 4 sowie 2 und 3 der $\mathrm{Cp}$-Liganden durch das Substitutionsmuster und der daraus resultierenden Prochiralität des zentralen Kohlenstoffatoms $C(6)$ diastereotop (s. Tab. 3),

So findet man im ${ }^{1} \mathrm{H}$-NMR-Spektrum des Tosylates 7 fünf Signale für die Methylprotonen der $\mathrm{Cp}^{*}$-Liganden. Im Spektrum des Alkohols 2 und des Chlorformyl-Derivates $\mathbf{6}$ weisen die Methylgruppen an $\mathrm{C}(2)$ und $\mathrm{C}(3)$ eine zufällige Isochronie auf. Entsprechend beobachtet man hier jeweils nur vier Resonanzen für die Methylgruppen der Cp*-Liganden. In 2 wird die Resonanz des Methinprotons am zentralen Kohlenstoffatom durch Kopplung mit dem Proton der Hydroxy-Gruppe zum Dublett aufgespalten. Auffällig ist die vergleichsweise geringe Kopplungskonstante von nur $3.9 \mathrm{~Hz}$ (zum Vergleich: die entsprechende Kopplungskonstante im Diels-Alder-Produkt 3 beträgt ca. $11 \mathrm{~Hz}$ ). Wir führen dies auf die geometrische Verzerrung am zentralen Kohlenstoffatom zurück. Offensichtlich wird der Bindungswinkel $\mathrm{H}-\mathrm{C}(6)-\mathrm{OH}$ zur Kompensation der Aufweitung des Win- 
kels $\mathrm{Cp} \mathrm{p}^{*}-\mathrm{C}(6)-\mathrm{Cp}$ * relativ zum idealen Tetraederwinkel deutlich verringert.

Tab. 3. NMR-Daten von 2, 6 und 7

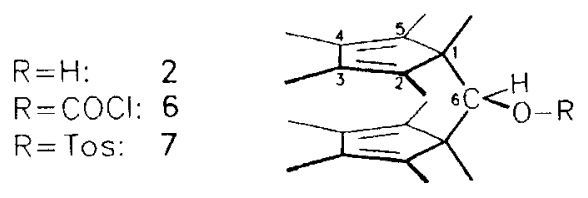

\begin{tabular}{|c|c|c|c|c|c|c|c|}
\hline & ${ }^{1} \mathrm{H}-\mathrm{NMR}$ & $\left(\mathrm{C}_{6} \mathrm{D}_{6}\right.$ & & & ${ }^{13} \mathrm{C}-\mathrm{I}$ & SMR & $\left.\mathrm{C}_{6} \mathrm{D}_{6}\right)$ \\
\hline & 2 & 6 & 7 & & 2 & 6 & 7 \\
\hline 2,5-Me & 1.73 & 1.80 & 1.88 & $C-2-5$ & 134.6 & 135.9 & 135.4 \\
\hline & 1.88 & 1.58 & 1.63 & & 135.7 & 136.1 & 135.8 \\
\hline 3,4-Me & 1.66 & 1.48 & 1.52 & & 139.2 & 136.6 & 137.3 \\
\hline & & & 1.49 & & 142.0 & 138.7 & 139.4 \\
\hline 1-Me & 1.10 & 0.91 & 1.15 & C-1 & 61.6 & 59.6 & 60.2 \\
\hline $\mathrm{Ar}-\mathrm{Me}$ & - & - & 1.79 & C-6 & 78.9 & 91.2 & 93.2 \\
\hline $6-\mathrm{H}$ & 3.90 & 5.44 & 5.49 & 2,5-Me & 11.0 & 11.0 & 11.1 \\
\hline & $\mathrm{d}(J=3.9 \mathrm{~Hz})$ & & & & 11.2 & 11.4 & 11.7 \\
\hline $6-\mathrm{OH}$ & 1.28 & - & - & & 12.0 & 12.8 & 13.2 \\
\hline & $\mathrm{d}(J=3.9 \mathrm{~Hz})$ & & & & 12.4 & & \\
\hline$-\mathrm{C}_{6} \mathrm{H}_{4}-$ & - & - & 6.71 , & $1-\mathrm{Me}$ & 21.2 & 20.7 & 22,9 \\
\hline & & & 7.89 & $\mathrm{Ar}-\mathrm{Me}$ & - & - & 21.0 \\
\hline & & & & $-\mathrm{C}_{6} \mathrm{H}_{4}-$ & - & - & 129.4 \\
\hline & & & & & & & $\begin{array}{l}137.9 \\
143.5\end{array}$ \\
\hline & & & & $\mathrm{CO}$ & - & 151.2 & - \\
\hline
\end{tabular}

In den Estern 6 und 7 tritt die Resonanz des MethinProtons im Vergleich zu 2 auffällig Tieffeld-verschoben bei $\delta=5.5$ auf. Hierfür ist offensichtlich der stark elektronenziehende Einfluß der jeweiligen Esterfunktion ursächlich. Dieser Effekt spiegelt sich auch in der chemischen Verschiebung des Zentralatoms in 6 und $7 \mathrm{im}{ }^{1.3} \mathrm{C}-\mathrm{NMR}$-Spektrum wider. So tritt das Signal dieses Kohlenstoffatoms Tieffeldverschoben bei $\delta \approx 90$ auf.

\section{Experimenteller Teil}

Alle Arbeiten wurden unter Ausschluß von Luft und Feuchtigkeit unter Argon durchgeführt; verwendete Geräte, Chemikalien und Lösungsmittel waren entsprechend vorbereitet. - Schmelzpunktc: Büchi-510-Schmelzpunktbestimmungsapparatur, Probenröhrchen abgeschmolzen. - NMR (Bruker AM 300): ${ }^{1} \mathrm{H}-\mathrm{NMR}(300.1 \mathrm{MHz})$ ext. TMS, ${ }^{13} \mathrm{C}\left\{{ }^{1} \mathrm{H}\right\}$-NMR (75.4 MHz) ext. TMS. - MS: Varian $\mathrm{CH}$ $5(70 \mathrm{eV}, 300 \mu \mathrm{A}$ Emission); cs sind nur charakteristische FragmentIonen angegeben. - IR: Perkin Elmer 598 Infrared Spectrometer. - CH-Analysen: Mikroanalytisches Laboratorium Beller, Göttingen; Mikroanalytisches Laboratorium der Universität Bielefeld.

Bis (1,2,3,4,5-pentamethyl-2,4-cyclopentadien-1-yl)methanol (2): $0.53 \mathrm{~g}(13.9 \mathrm{mmol}) \mathrm{LiAlH}_{4}$ werden in $150 \mathrm{ml}$ THF suspendiert. Hierzu tropft man bei $-30^{\circ} \mathrm{C}$ eine Lösung von $4.00 \mathrm{~g}(13.4 \mathrm{mmol})$ Bis(pentamethylcyclopentadienyl)keton $(1)^{[6]}$ in $50 \mathrm{ml}$ Diethylether und rührt $12 \mathrm{~h}$. Anschließend wird bei dieser Temp. vorsichtig mit Eis hydrolysiert und mit konz. $\mathrm{HCl}$ angesäuert. Die organische Phase wird dekantiert und mit $\mathrm{Na}_{2} \mathrm{CO}_{3}$ neutralisiert. Man trocknet mit $\mathrm{MgSO}_{4}$ und destilliert die Solvenzien i. Vak. ab. Der Rückstand wird in $40 \mathrm{ml}$ Pentan aufgenommen. Nach Zugabe von $2 \mathrm{ml}$ Pyridin kristallisiert Verbindung 2 bei $-70^{\circ} \mathrm{C}$ als Pyridin-Addukt in farblosen Rhomben. Zur Entfernung des Pyridins löst man das Addukt in Hexan und destilliert die flüchtigen Bestandteile i. Vak. ab. Dieser
Vorgang wird mehrmals wiederholt. Ausb. $2.81 \mathrm{~g} \mathrm{(70 \% )} \mathrm{2,} \mathrm{Schmp.}$ $102^{\circ} \mathrm{C}$. - MS, $m / \varepsilon(\%): 300(11)\left[\mathrm{M}^{+}\right], 165$ (3), $150(3), 136(73)$, 135 (100). - IR (KBr): $\tilde{v}=3520 \mathrm{~cm}^{-1}(\mathrm{OH}) .-\mathrm{C}_{21} \mathrm{H}_{32} \mathrm{O}(300.5):$ ber. C 83.94, H 10.73; gef. C 83.87, H 10.88 .

$1,2,4,5,6,7,8,9,10,11$-Decamethyltetracyclo $\left[5.3 .1 .0^{2.8} .0^{4.1 t}\right]$ undeca5,9-dien-3-ol (3): Eine Lösung von $0.50 \mathrm{~g}(1.68 \mathrm{mmol})$ des Alkohols 2 in Hexan wird $12 \mathrm{~h}$ bei Raumtemp. gerührt. Anschließend wird das Solvens i. Vak. abdestilliert und der tetracyclische Alkohol 3 als farbloses Pulver erhalten. Ausb. quantitativ, Diastereomerengemisch, Schmp. $102^{\circ} \mathrm{C}$.

Hauptisomer: ${ }^{1} \mathrm{H}-\mathrm{NMR}\left(\mathrm{C}_{6} \mathrm{D}_{6}\right): \delta=0.60\left(\mathrm{~s}, 3 \mathrm{H}, \mathrm{CH}_{3}\right), 0.82(\mathrm{~s}, 3 \mathrm{H}$, $\left.\mathrm{CH}_{3}\right), 0.87$ (s, $\left.3 \mathrm{H}, \mathrm{CH}_{3}\right), 0.88\left(\mathrm{~s}, 3 \mathrm{H}, \mathrm{CH}_{3}\right), 0.89\left(\mathrm{~s}, 3 \mathrm{H}, \mathrm{CH}_{3}\right), 1.11$ $\left(\mathrm{s}, 3 \mathrm{H}, \mathrm{CH}_{3}\right), 1.41\left(\mathrm{~s}, 6 \mathrm{H}, \mathrm{CH}_{3}\right), 1.44\left(\mathrm{~s}, 3 \mathrm{H}, \mathrm{CH}_{3}\right), 1.46\left(\mathrm{~s}, 3 \mathrm{H}, \mathrm{CH}_{3}\right)$, $3.33\left(\mathrm{~d},{ }^{3} J=11.3 \mathrm{~Hz}, 1 \mathrm{H},-\mathrm{CH}-\right) .-{ }^{13} \mathrm{C}-\mathrm{NMR}\left(\mathrm{C}_{6} \mathrm{D}_{6}\right): \delta=10.5$ $\left(\mathrm{C}-\mathrm{CH}_{3}\right), 11.8\left(\mathrm{C}-\mathrm{CH}_{3}\right), 12.4\left(\mathrm{C}-\mathrm{CH}_{3}\right), 12.6\left(\mathrm{C}-\mathrm{CH}_{3}\right), 13.0$ $\left(\mathrm{C}-\mathrm{CH}_{3}\right), \quad 13.3\left(\mathrm{C}-\mathrm{CH}_{3}\right), \quad 14.6\left(\mathrm{C}-\mathrm{CH}_{3}\right), 15.9\left(\mathrm{C}-\mathrm{CH}_{3}\right), 16.7$ $\left(\mathrm{C}-\mathrm{CH}_{3}\right), \quad 19.2\left(\mathrm{C}-\mathrm{CH}_{3}\right), \quad 55.3\left(\mathrm{C}-\mathrm{CH}_{3}\right), \quad 59.7\left(\mathrm{C}-\mathrm{CH}_{3}\right), 61.7$ $\left(\mathrm{C}-\mathrm{CH}_{3}\right), 62.6\left(\mathrm{C}-\mathrm{CH}_{3}\right), 65.3\left(\mathrm{C}-\mathrm{CH}_{3}\right), 69.4\left(\mathrm{C}-\mathrm{CH}_{3}\right), 84.0(\mathrm{C}-\mathrm{OH})$, $133.1\left(\mathrm{C}-\mathrm{CH}_{3}\right), 135.9\left(\mathrm{C}-\mathrm{CH}_{3}\right), 136.7\left(\mathrm{C}-\mathrm{CH}_{3}\right), 139.7\left(\mathrm{C}-\mathrm{CH}_{3}\right)$. MS, $m / z(\%): 300(11)\left[\mathrm{M}^{\prime}\right], 165(3), 150(3), 136(73), 135$ (100). IR (KBr): $\tilde{v}=3520 \mathrm{~cm}^{-1}(\mathrm{OH}) .-\mathrm{C}_{21} \mathrm{H}_{32} \mathrm{O}$ (300.5): ber. C 83.94, H 10.73; gef. C 83.86, H 10.88 .

Bis( 1,2,3,4,5-pentamethyl-2,4-cyclopentadien-1-yl)methyl-chloroformiat (6): Eine Lösung von $0.50 \mathrm{~g}(1.68 \mathrm{mmol})$ des Alkohols 2 in $30 \mathrm{ml}$ Diethylether wird bei $-30^{\circ} \mathrm{C}$ mit $1.05 \mathrm{ml}$ einer $1.60 \mathrm{M}$ Lösung von $n$-Butyllithium in Hexan (1.68 mmol) versetzt. Man rührt noch $2 \mathrm{~h}$ und tropft anschließend $1.00 \mathrm{ml}$ einer $1.93 \mathrm{M}$ Lösung von Phosgen in Toluol $(1.93 \mathrm{mmol})$ zu. Nach $12 \mathrm{~h}$ werden die flüchtigen Bestandteile i.Vak. entfernt, und der Rückstand wird zweimal in der Kälte mit je $30 \mathrm{ml}$ Hexan extrahiert. Anschließend entfernt man das Lösungsmittel i. Vak. 6 fällt dabei als farbloses Pulver an. Kristallisation aus Hexan bei $-70^{\circ} \mathrm{C}$ liefert farblose Plättchen. Ausb. 0.50 g $(83 \%)$, Schmp. 73 C, MS, $m / z\left(\%\right.$, bez. auf $\left.{ }^{35} \mathrm{Cl}\right): 361(10)$ $\left[\mathrm{M}^{+}\right], 282(4), 147(100), 135(36), 134$ (74). $-\mathrm{C}_{22} \mathrm{H}_{31} \mathrm{ClO}_{2}(363.0)$ : ber. C 72.80, H 8.61 ; gef. C 73.10, H 8.55 .

Bis (1,2,3,4,5-pentamethyl-2,4-cyclopentadien-1-yl)methyl-p-to-

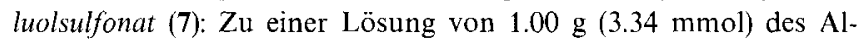
kohols 2 in $50 \mathrm{ml}$ Diethylether werden bei $-30 \mathrm{C} 2.40 \mathrm{ml}$ eincr $1.60 \mathrm{M}$ Lösung von $n$-Butyllithium in Hexan getropft. Man rührt

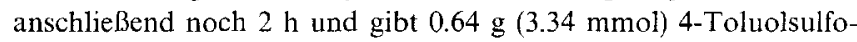
nylchlorid zu. Die Reaktionsmischung wird $12 \mathrm{~h}$ gerührt. Die Solvenzien werden in der Kälte i. Vak. abdestilliert. Man wäscht den farblosen Rückstand dreimal mit kaltem Hexan und trocknet in der Kälte i. Hochvak. 7 wird als farbloses Pulver im Gemisch mit Lithiumchlorid isoliert. Ausb. (bezogen auf 7): ca. $1.2 \mathrm{~g}$ (ca. $80 \%$ ). - MS, $m / z(\%): 454(20)\left[\mathrm{M}^{+}\right], 319(8), 282(10), 267(9), 147(50)$, $135(100)$.

Pentacyclus 9: $0.50 \mathrm{~g}$ des Tosylates 7 werden in $30 \mathrm{ml}$ THF suspendiert. Man versetzt die Mischung mit $10 \mathrm{ml}$ einer ges. $\mathrm{NaHCO}_{3^{-}}$ Lösung und erhitzt kurz bis zum Rückfluß. Nach Abkühlen wird die Reaktionslösung zweimal mit $20 \mathrm{ml}$ Hexan extrahiert. Man trocknet mit $\mathrm{MgSO}_{4}$ und entfernt die Solvenzien i.Vak. 9 fällt als gelbes Pulver an. Ausb. quantitativ, Schmp. $68^{\circ} \mathrm{C}-{ }^{1} \mathrm{H}-\mathrm{NMR}$ $\left(\mathrm{C}_{6} \mathrm{D}_{6}\right): \delta=0.59,0.67,0.70,0.75,1.04,1.08,1.09\left(7 \mathrm{~s}, 7 \times 3 \mathrm{H}, \mathrm{CH}_{3}\right)$, $1.46,1.48\left(2 \mathrm{q},{ }^{5} J_{\mathrm{HH}}=1.3 \mathrm{~Hz}, 2 \times 3 \mathrm{H}, \mathrm{CH}_{3}\right), 4.72,4.84\left(2 \mathrm{~d},{ }^{2} J=1\right.$ $\left.\mathrm{Hz}, 2 \times 1 \mathrm{H},=\mathrm{CH}_{2}\right) .-{ }^{13} \mathrm{C}-\mathrm{NMR}\left(\mathrm{C}_{6} \mathrm{D}_{6}\right): \delta=9.6,10.6,11.4,12.3$, 12.5, 13.0, 13.5, $14.4\left(9 \mathrm{CH}_{3}\right), 39.9(-\mathrm{CH}-), 41.7,45.6,57.3,59.5$, 59.9, 65.6, 74.9 (quart. Brückenkopf-C), $96.3\left(\mathrm{C}=\mathrm{CH}_{2}\right), 130.9,136.8$ (2 C=C Ring), $160.5\left(C=\mathrm{CH}_{2}\right)$. - MS $m / z(\%): 282(13)\left[\mathrm{M}^{+}\right], 147$ (100), 134 (40). $-\mathrm{C}_{21} \mathrm{H}_{30}$ (282.5): ber. C 89.30, H 10.70; gef. C 89.43, H 10.79 . 
[1] P. Jutzi, D. Kanne, C. Krüger, Angew. Chem. 1986, 98, 163-164; Angew. Chem. Int. Ed. Engl. 1986, 25, 164-165.

[2] P. Jutzi, F. X. Kohl, P. Hofmann, C. Krüger, Y.-H. Tsay, Chem. Ber. 1980, 113, 757-769.

${ }^{[3]}$ L. Fernhold, A. Haaland, P. Jutzi, F. X. Kohl, R. Seip, Acta Chem. Scand., Ser. A, 1984, 38, 211-216.

${ }^{[4]}$ J. L. Atwood, W. E. Hunter, J. Chem. Soc., Chem. Commun. $1981,925-927$.

${ }^{[5]}$ F. X. Kohl, P. Jutzi, Chem. Ber. 1987, 120, 1539-1543

${ }^{[6]}$ P. Jutzi, K.-H. Schwartzen, A. Mix, H.-G. Stammler, B. Neumann, Chem. Ber. 1993, 126, 415-420.

${ }^{[7]}$ P. Jutzi, B. Krato, M. Hursthouse, Chem. Ber. 1987, 120, 565-574; P. Jutzi, D. Kanne, M. Hursthouse, A. J. Howes, ibid. 1988, 121, 1299-1305; A. J. Bard, A. H. Cowley, J. K. Leland, G. J. N. Thomas, N. C. Norman, P. Jutzi, C. P. Morley, E. Schlüter, J. Chem. Soc., Dalton Trans. 1985, 1303-1307.

${ }^{[8]}$ R. J. Bushby, D. W. Jones, J. Chem. Soc., Chem. Commun. 1979, $688-690$.

${ }^{[9]}$ D. Fejtler, G. M. Whitesides, Inorg. Chem. 1976, 15, 466-469.

${ }^{{ }^{10]}}$ Bei der Erzeugung des $\mathrm{Cp}^{*}$-Kations in Gegenwart von 2,6-Lutidin wird aus sterischen Gründen ebenfalls kein $N$-Cp*-substituiertes Pyridin-Derivat erhalten: P. Jutzi, A. Mix, Chem. Ber. $1992,125,951-954$.

${ }^{1113}$ Brune et al. erhielten derartige Cyclen durch Erhitzen von (Pentamethylcyclopentadienyl)methansulfonaten: H.-A. Brune, P.
Lach, G. Schmidtberg, Chem. Ber. 1985, 118, $2671-2680$; H.A. Brune, P. Lach, G. Schmidtberg, ibid. 1985, 118, $2681-2691$. ${ }^{[12]}$ L. De Vries, J. Am. Chem. Soc. 1960, 82, 5242-5244.

${ }^{[13]}$ Siehe hierzu auch L. A. Paquette, G. R. Krow, Tetrahedron Lett. $1968,17,2139-2142$.

${ }^{[14]} \mathrm{S}$. Winstein, M. Battiste, J. Am. Chem. Soc. 1960, 82, $5244-5245$

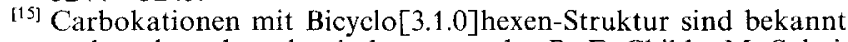
und auch spektroskopisch untersucht: R. F. Childs, M. Sakai, B. D. Parrington, S. Winstein, J. Am. Chem. Soc. 1974, 96, 6403-6409.

${ }^{[16]}$ R. F. Childs, S. Winstein, J. Am. Chem. Soc. 1974, 96, $6409-6417$.

${ }^{117]} \mathrm{Cp}^{*}$ OTos ist vermutlich äußerst thermolabil; man vergleiche mit der Thermolabilität der Cp*-Halogenide: P. Jutzi, K.-H. Schwartzen, A. Mix, Chem. Ber. 1990, 123, 837-840.

${ }^{[18]}$ Weitere Einzelheiten zur Kristallstrukturanalyse von 2 und 6 können beim Fachinformationszentrum Karlsruhe, Gesellschaft für wissenschaftlich-technische Information mbH, D-76344 Eggenstein-Leopoldshafen, unter Angabe der Hinterlegungsnummer CSD-57754, der Autorennamen und des Zeitschriftenzitats angefordert werden.

$[125 / 93]$ 